\title{
Parameter Selection for the Supply System in a Pilger Mill
}

\author{
Yu. B. Chechulin ${ }^{a}$, Yu. A. Popov ${ }^{a}$, S. N. Filatov ${ }^{b}$, and B. Ya. Sokolinskii ${ }^{b}$ \\ ${ }^{a}$ Yeltsin Ural Federal University, Yekaterinburg, Russia \\ ${ }^{b}$ OAO ChTPZ, Chelyabinsk, Russia
}

\begin{abstract}
The braking chamber in the supply system of a Pilger pipe mill is modernized. The influence of constant and variable parameters of the supply system on braking is studied.
\end{abstract}

Keywords: Pilger mill, supply system, hydraulic braking chamber, annular gap, choking

DOI: $10.3103 / \mathrm{S} 0967091213120048$

Pipe-rolling systems with a Pilger mill are used to produce seamless thin-walled large-diameter pipe from carbon and alloy steel. The rollers in the working cell rotate here in the opposite direction to blank supply. On the input side of the Pilger mill, the most important component is the supply system for the pipe blank, whose basic function is to supply the sleeve to the roller throat and rotate it around its axis by a specified angle. The supply operations must be synchronous with roller rotation. That calls for soft braking and stopping in a strictly specified position after a certain interval. The mill operation and the overall speed of pipe production largely depend on the reliability of the supply system.

In existing mills, the supply system has a pneumatic drive. Various methods may be used for braking: pneumatic, hydraulic, or mechanical. Recently, supply systems with hydraulic braking have been widely adopted. They consist of an air chamber containing a piston and a hydraulic chamber containing the braking bush and piston. The pistons are attached to a common rod, which passes through both chambers and is connected at one end to the piston of the air chamber and at the other to the mandrel head.

Consider the supply system in Pilger mill 2 at OAO ChTPZ, which employs mechanical braking (Fig. 1). The system includes a housing with a built-in pneumatic cylinder, a braking chamber, a drill connected to the piston, and a ratchet and pawl system. The drill is mounted in guide bushes and may rotate around its longitudinal axis. The braking chamber consists of a set of plate strings retained on one side by a damper and on the other by a flanged lid.

This design has certain problems. Since the system experiences impacts in the course of operation and the dynamic load on its components is high, the springs of the braking chamber rapidly fail. In addition, the springs do not always undergo appropriate heat treatment, which reduces their reliability and accelerates their failure. After spring failure, the dynamic load is transmitted to the housing of the air piston and the flanged lid, resulting in periodic fracture. It is also impossible to control the braking process. The traditional pneumohydraulic system contains three pistons attached to a single long shaft, in which elastic deformation and stability loss produce an edge effect and rapid wear at contact of the piston with the cylindrical guide surfaces.

To improve the performance of this system, the spring-based braking chamber may be replaced by a hydraulic chamber with a floating piston. That eliminates the noted defects and permits control of the braking as a function of the pipe blank's dimensions. The working fluid in the braking chamber is water, whose specific heat is less than that of oil, with consequent improvement in heat extraction.

In Fig. 2, we show the modernized hydraulic chamber. In the chamber, a braking bush rests on the rear bearing on one side and is supported by the flanged lid on the other side. The hydraulic piston,

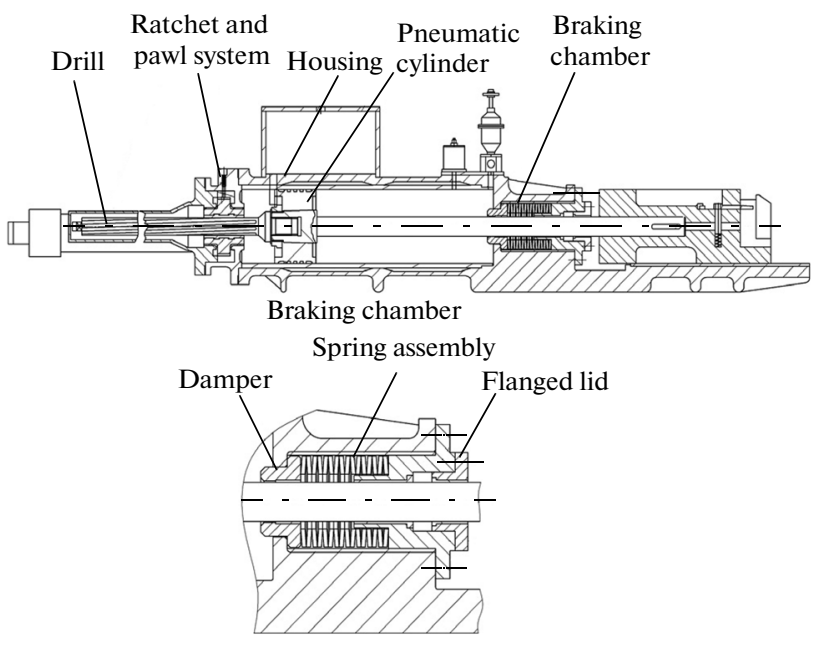

Fig. 1. Pneumomechanical supply system. 
with a shorter path than the pneumatic piston, takes the form of a bush capable of free motion along the shaft in the supply system. In the return path of the shaft, contact of the air piston with the piston bush of the hydraulic piston separates the supply period into stages of acceleration and deceleration of the moving masses, which include the clamp for the pipe blank (the mandrel head), the mandrel, and the pipe blank. Braking is due to the hydraulic drag in liquid motion through an annular gap and the choking aperture. This design, for which a patent application is pending, has been successfully used in practice.

For this braking system, we need to determine the constant parameters of the braking chamber (specified in the design) and the variable parameters used in adjusting the process in accordance with the pipe being rolled. The acceleration from the extreme rear position is due to compressed air in the pneumatic chamber. In this stage, we may write the equation of motion in the form

$$
m_{\mathrm{mo}} \ddot{x}-p_{0} F_{1}\left(\frac{H}{H-h+x}\right)^{k}+R_{\mathrm{fr}}=0,
$$

where $m_{\mathrm{mo}}$ is the mass of the mobile components and the sleeve; $F_{1}$ is the active area of the air piston; $p_{0}$ is the initial air pressure in the pneumatic chamber; $H$ is the length of the air space in the extreme forward piston position; $h$ is the distance traveled by the piston during rolling (the piston path); $R_{\mathrm{fr}}$ is the frictional force; $k$ is the adiabatic index for the air.

From Eq. (1), we find the acceleration time and final speed, which serves as the initial condition for the braking stage.

In active hydraulic braking of the piston moving along the braking bush, with known dimensions of the braking chamber, the equation of motion takes the form

$$
m_{\mathrm{mo}} \ddot{x}-p_{0} F_{1}\left(\frac{H}{H-l+x}\right)^{k}-p_{\mathrm{h}} F_{2}-R_{\mathrm{fr}}=0,
$$

where $p_{\mathrm{h}}$ is the pressure in the hydraulic chamber during braking

$$
p_{\mathrm{h}}=\frac{12 \mu Q l^{\prime}}{b(\delta+f / b)^{3}}+\frac{\rho Q^{2}}{2 b^{2}(\delta+f / b)^{2}} .
$$

Here $Q=F_{2} \ddot{x}$ is the total liquid flow rate through the annular gap and the choking apertures; $\mu$ is the dynamic viscosity of the liquid; $l^{\prime}$ is the length of the annular gap, determined either by the length of the hydraulic piston or by the chamber length; $F_{2}$ is the current area of the annular gap; $\delta$ is the dimension of the annular gap; $f$ is the total area of the choking apertures; $b$ is the length of the annular slot $(b=\pi D)$.

The first term in Eq. (3) is the pressure loss due to friction in the liquid; the second corresponds to the

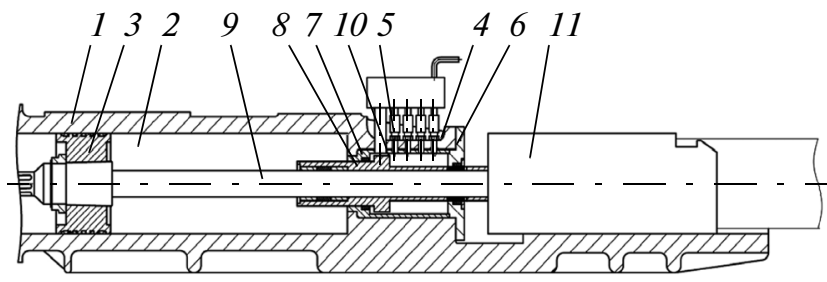

Fig. 2. Modernized braking chamber: (1) housing of supply system; (2) air chamber; (3) air piston; (4) hydraulic chamber; (5) choking aperture; (6) flanged lid; (7) braking bush; (8) hydraulic piston.

hydrodynamic losses. Substituting Eq. (3) into Eq. (2), we obtain

$$
\begin{gathered}
m_{\mathrm{mo}} \hat{x}+p_{0} F_{1}\left(\frac{H}{H-l+x}\right)^{k} \\
-\frac{12 \mu F_{2}^{2} \ddot{x} l^{\prime}}{b(\delta+f / b)^{3}}-\frac{\rho F_{2}^{3} \dot{x}^{2}}{2 b^{2}(\delta+f / b)^{2}}-R_{\mathrm{fr}}=0 .
\end{gathered}
$$

We consider the acceleration and braking stages with the following parameter values.

The constant parameters are as follows:

Active area of air piston $F_{1}, \mathrm{~m}^{2}$

Length of the air space in the extreme

forward piston position $H, \mathrm{~m}$

Piston path $h, \mathrm{~m}$

Adiabatic index for air $k$

1.4

Area of hydraulic piston $F_{2}, \mathrm{~m}^{2}$

0.101

Density of working liquid (water) $\rho, \mathrm{kg} / \mathrm{m}^{3}$

1000

Dynamic viscosity of water $\mu, \mathrm{Pa} s$

0.01

Diameter, $\mathrm{m}$ :

hydraulic piston $D_{\mathrm{h}}$

0.437

supporting shaft $D_{\mathrm{sh}}$

0.250

initial diameter of hydraulic bush

0.460

at initial cone $D_{0}$

final diameter of hydraulic bush at initial cone $D_{1}$

diameter of hydraulic bush in cylindrical

section $D$

Length of braking chamber $l, \mathrm{~m}$

Frictional force $R_{\mathrm{fr}}, \mathrm{N}$

0.446

0.439

0.355

5000

The variable parameters are as follows:

Mass of mobile components $m_{\mathrm{mo}}, \mathrm{kg}$

8000,10000 , 12000

Initial air pressure in pneumatic chamber

$1.2-2.0$

$p_{0}, 10^{5} \mathrm{~Pa}$

Number of rows of apertures in the braking chamber $n$

No more

Number of holes per row $\left(n_{1}-n_{4}\right)$

than 4

No more

than 2

Diameter of choking apertures $d_{0}, \mathrm{~mm}$
No more

than 30 


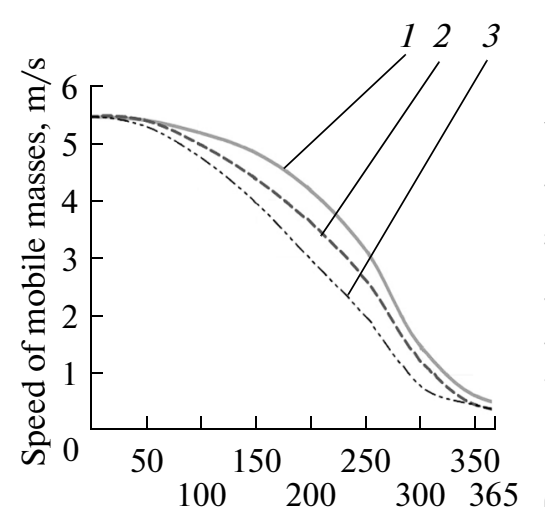

Distance traveled, $\mathrm{mm}$

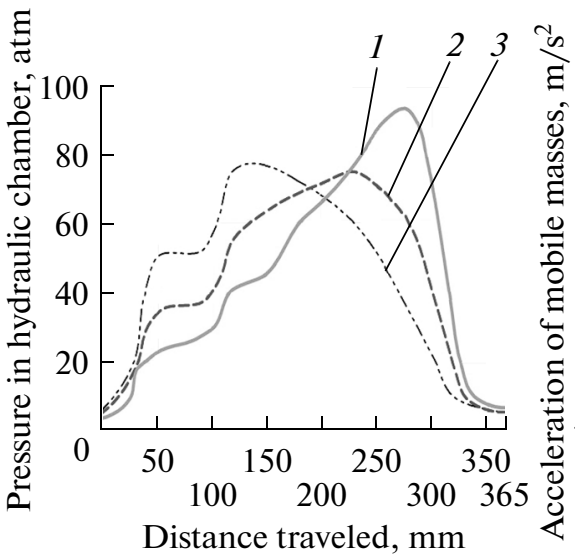

Distance traveled, $\mathrm{mm}$

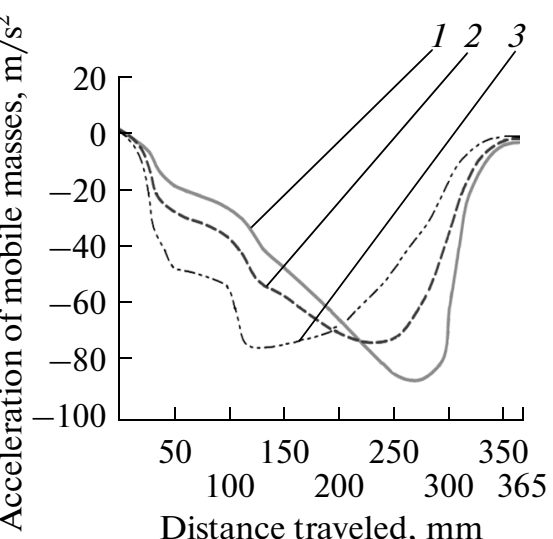

Distance traveled, $\mathrm{mm}$

Fig. 3. Influence of the initial size of the annular gap in the hydraulic chamber on braking with an initial gap of 5.5 (1), 4.5 (2), and $3.5(3) \mathrm{mm}$.

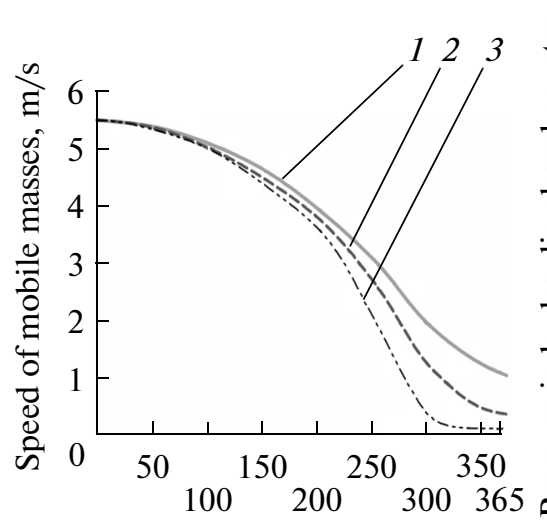

Distance traveled, $\mathrm{mm}$

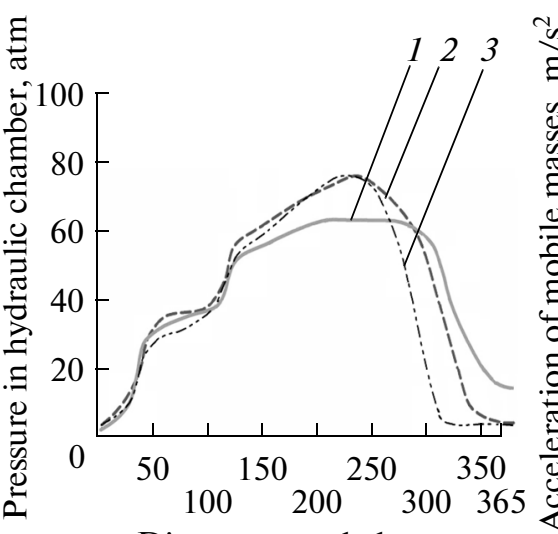

Distance traveled, $\mathrm{mm}$

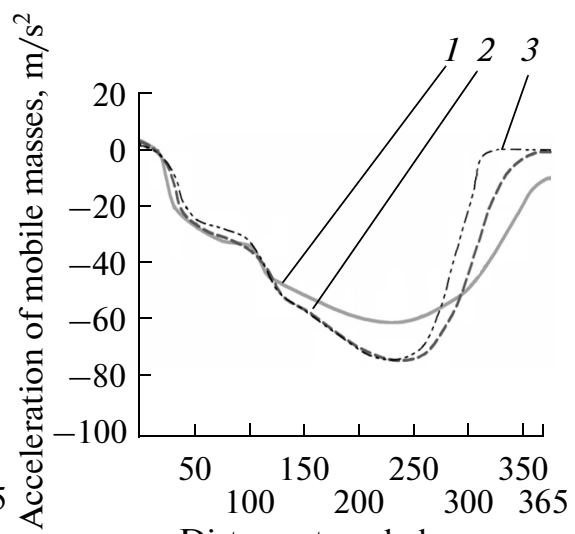

Distance traveled, $\mathrm{mm}$

Fig. 4. Influence of the final size of the annular gap in the hydraulic chamber on the braking process: (1) $1.5 \mathrm{~mm}$; (2) $1.0 \mathrm{~mm}$; (3) $0.5 \mathrm{~mm}$.

It is important to select the minimum speed of the shaft at the end of its return path and the maximum pressure in the braking chamber, as well as the maximum acceleration of the shaft. We now consider the solution of Eq. (4) when $m_{\mathrm{mo}}=10000 \mathrm{~kg}$, with different dimensions of the braking bush and with different choking. The other parameters are as already noted. The initial pressure in the air chamber is $1.5 \mathrm{~atm}$. (This is the minimum value at which the total acceleration and deceleration time is no more than the permissible value of $0.5 \mathrm{~s}$.)

In Fig. 3, we show the influence of the initial size of the annular gap in the hydraulic chamber on braking. This parameter has no significant influence on the final speed but does affect the peak pressure and acceleration and the position of these peaks. We assume the choking configuration 2-1-0-0 (with two open holes in the first row, one in the second, and none in the third and fourth rows); the hole diameter is $30 \mathrm{~mm}$. With the specified constant parameters, the best choice for the initial size of the annular gap is $4.5 \mathrm{~mm}$.
In Fig. 4, we show the influence of the final size of the annular gap on the braking process. Its influence on the final velocity is significant, but it has considerable less influence on the maximum pressure and acceleration. We find that reducing the final size of the annular gap from 1.5 to $0.5 \mathrm{~mm}$ reduces the final shaft speed by a factor of six, with increase in the maximum pressure and deceleration by $20 \%$. If the final size of the annular gap is $0.5 \mathrm{~mm}$, we may reduce the length of the braking chamber from 365 to $300 \mathrm{~mm}$, since further motion occurs at constant speed, but this significantly increases the braking time. We assume the choking configuration $2-1-0-0$ (with two open holes in the first row, one in the second, and none in the third and fourth rows).

In Fig. 5, we show the influence of choking on the braking characteristics. The initial and final sizes of the annular gap are assumed to be 4.5 and $0.5 \mathrm{~mm}$. To elucidate the variation in the braking characteristics with variation of the choking, we assume specific posi- 


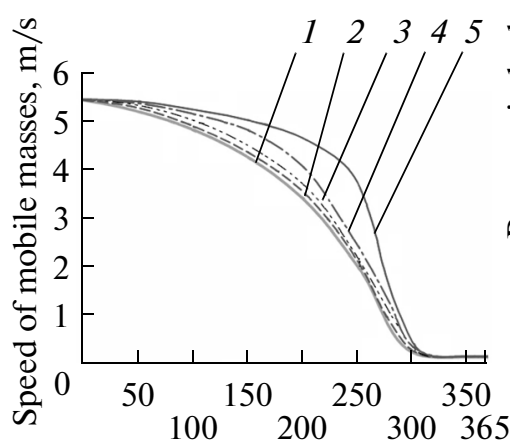

Distance traveled, $\mathrm{mm}$

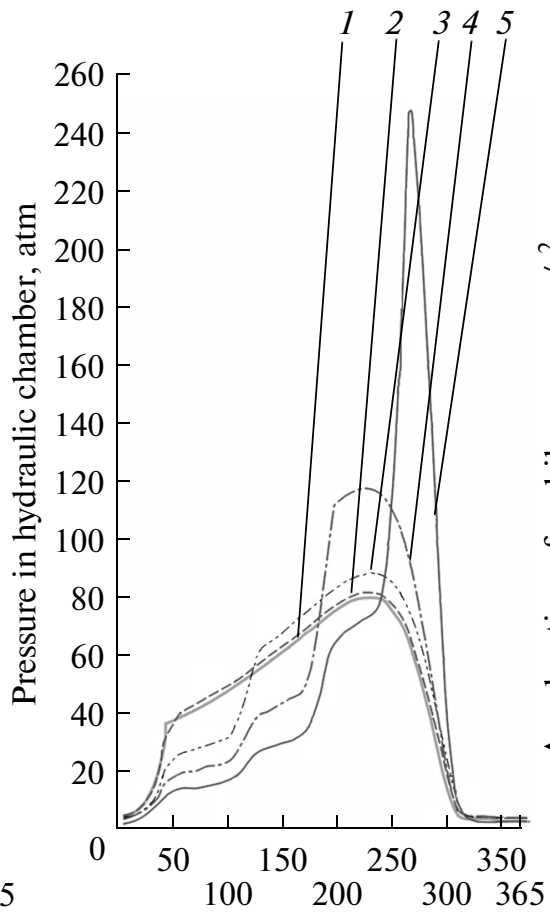

Distance traveled, $\mathrm{mm}$

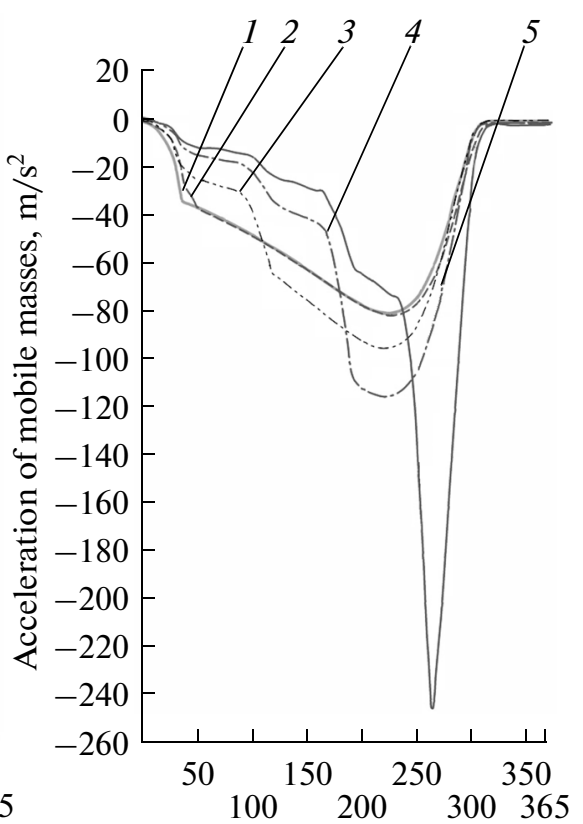

Distance traveled, $\mathrm{mm}$

Fig. 5. Influence of the choking configuration on the braking process: (1) $0-0-0-0$; (2) $2-0-0-0$; (3) $2-2-0-0$; (4) $2-2-2-0$; (5) $2-2-2-2$.

tions of the choking holes. We obtain the following results.

(1) Choking does not affect the final braking speed.

(2) Uniform opening of the choking holes over the whole length of the braking bush reduces the initial pressure front in the braking chamber. However, its value does not exceed half of the subsequent maximum value.

(3) With increase in the number of rows of open holes over the length of the braking bush, there is significant increase in the maximum pressure and acceleration.

(4) For stable rolling, additional choking in the first third of the piston bush's path in the hydraulic chamber is best.
Adjustment of the acceleration and deceleration parameters permits optimal operation of the supply system with blanks of different mass.

\section{CONCLUSIONS}

A new design is proposed for the supply system of a Pilger mill with a shorter shaft and braking chamber. That increases the longitudinal stability of the shaft, reduces the size of the supply system, and reduces the mass and dynamic load of the mobile components. We have developed an algorithm and program for calculating the optimal parameters of the supply system.

Translated by Bernard Gilbert 